[Chem. Pharm. Bull.

35( 9 ) 3726-3733(1987) $]$

\title{
Metabolism of Paeoniflorin and Related Compounds by Human Intestinal Bacteria. II. ${ }^{1)}$ Structures of $7 S$ - and $7 R$-Paeonimetabolines I and II Formed by Bacteroides fragilis and Lactobacillus brevis
}

\author{
Yue-Zhong Shu, ${ }^{a}$ Masao Hattori, ${ }^{a}$ Teruaki Akao, ${ }^{b}$ Kyolchi Kobashi, ${ }^{b}$ \\ Katsumi Kagei, ${ }^{c}$ KeIICHi FuKuyama, ${ }^{c}$ TOMitake Tsukihara ${ }^{c}$ \\ and TSUNEO NAMBA*,a
}

\author{
Research Institute for Wakan-Yaku (Oriental Medicines), ${ }^{a}$ Faculty of Pharmaceutical \\ Sciences, ${ }^{b}$ Toyama Medical and Pharmaceutical University, 2630 Sugitani, \\ Toyama 930-01, Japan and Faculty of Engineering, Tottori University, ${ }^{c}$ \\ 101 Minami 4-chome, Koyama-cho, Tottori 680, Japan
}

(Received February 25, 1987)

\begin{abstract}
Paeoniflorin from peony roots was incubated with a cell suspension of Bacteroides fragilis or Lactobacillus brevis isolated from human intestinal flora. Paeoniflorin was converted into the $7 S$ and $7 R$ isomers of paeonimetaboline $\mathrm{I}$ as major metabolites, along with the $7 R$ and $7 S$ isomers of paeonimetaboline II as minor metabolites in the case of the former strain. The structures were elucidated by various spectroscopic methods, and the structure of $7 R$-paeonimetaboline I was confirmed by X-ray analysis.
\end{abstract}

Keywords_-Bacteroides fragilis; biotransformation; 5-hydroxy-3,6-dimethyl-2,3-dihydrobenzofuran; intestinal bacteria; Lactobacillus brevis; Paeonia albiflora; paeoniflorin; paeonimetaboline I; paeonimetaboline II; X-ray analysis

In our preceding papers, ${ }^{2,3)}$ we reported that paeoniflorin (1), as well as oxypaeoniflorin and benzoylpaeoniflorin, isolated from peony roots, was converted into paeonimetabolines by human intestinal bacteria. The structure of a major metabolite was concluded to be $7 S$ paeonimetaboline I (2). Similarly, albiflorin, a minor constituent of the peony roots, was metabolized to paeonilactones A (10) and B.

In order to prepare a sufficient amount of paeonimetaboline I for further study, we again surveyed various bacterial strains from human feces for ability to transform $\mathbf{1}$ to $\mathbf{2}$ during a short period of incubation, and found that some bacterial strains such as Bacteroides fragilis and Lactobacillus brevis have potent transforming activity, which had not been noticed in the previous screening experiments because of the long period of incubation used (the metabolites decreased in amount during prolonged incubation and almost completely disappeared within $16 \mathrm{~h}){ }^{2)}$

In the present paper, we report the isolation of the new $7 R$-epimer (3) of paeonimetaboline I and a mixture of $7 R$ and $7 S$ isomers of paeonimetaboline II (4a and $\mathbf{4 b}$ ), and the elucidation of the structures of these metabolites by various spectroscopic methods.

\section{Materials and Methods}

Instruments - Melting points $(\mathrm{mp})$ were determined on a Yanagimoto micro-melting point apparatus and are uncorrected. Infrared (IR) spectra were measured with a Hitachi 260-10 infrared spectrophotometer. Proton and carbon-13 nuclear magnetic resonance $\left({ }^{1} \mathrm{H}-\mathrm{NMR}\right.$ and $\left.{ }^{13} \mathrm{C}-\mathrm{NMR}\right)$ spectra were measured with JEOL JNM-GX 400 $\left({ }^{1} \mathrm{H}, 400 \mathrm{MHz}\right)$, JEOL FX-270 $\left({ }^{1} \mathrm{H}, 270 \mathrm{MHz}\right)$ and JEOL-FX $90 \mathrm{Q}\left({ }^{1} \mathrm{H}, 90 \mathrm{MHz} ;{ }^{13} \mathrm{C}, 22.5 \mathrm{MHz}\right) \mathrm{NMR}$ spectrometers. 
In ${ }^{13} \mathrm{C}$-NMR spectra, the multiplicities were determined on the basis of the off-resonance decoupling (OFR) and insensitive nuclei enhanced by polarization transfer (INEPT) techniques. Tetramethylsilane was used as an internal standard in all the measurements. Mass spectra (MS) were measured with a JEOL JMS D-200 mass spectrometer at an ionization voltage of $70 \mathrm{eV}$. Specific rotations were taken on a Jasco model DIP-4 automatic polarimeter. Ultraviolet (UV) spectra were recorded on a Shimadzu UV-210A spectrophotometer. High-performance liquid chromatography (HPLC) was carried out on a Tri-Rotar-V equipped with a UVIDEC-100-V detector (JASCO) using a column ( $250 \mathrm{~mm} \times 4.6 \mathrm{~mm}$ i.d.) of Chemopak, Nucleosil 50-5 (5 $\mu$, Chemco Co. Ltd., Osaka).

Chromatography of Metabolites - Wakogel C-200 was used for column chromatography. Thin layer chromatography (TLC) was performed on Merck Kieselgel $60 \mathrm{~F}_{254}$ or Merck PSC-60 $\mathrm{F}_{254}$ (preparative) plates with a solvent system of $\mathrm{CHCl}_{3}-\mathrm{MeOH}$-benzene $(5: 1: 1)$. Spots on the plates were visualized by exposure to iodine vapor or by spraying with an anisaldehyde- $\mathrm{H}_{2} \mathrm{SO}_{4}$ reagent, followed by heating.

Metabolism of 1 by Lactobacillus brevis-A precultured bacterial suspension $(500 \mathrm{ml})$ of $L$. brevis was added to GAM broth $(4.5 \mathrm{l})$ and cultivated for $12 \mathrm{~h}$ at $37^{\circ} \mathrm{C}$ under anaerobic conditions. The culture was centrifuged at $7000 \mathrm{rpm}$ for $10 \mathrm{~min}$. The precipitates were washed with saline solution, centrifuged, and suspended in $0.1 \mathrm{M}$ phosphate buffer $(625 \mathrm{ml})$. The suspension was transferred into five tubes. Compound $1(600 \mathrm{mg} / 10 \mathrm{ml}$ in the same buffer) was then added portionwise into each tube and was anaerobically incubated for $4 \mathrm{~h}$ at $37^{\circ} \mathrm{C}$. The mixture was extracted three times with ethyl acetate (AcOEt, $200 \mathrm{ml}$ each) and the organic layer was concentrated in vacuo to give an oily residue. The combined residues $(0.3 \mathrm{~g})$ were applied to a column of silica gel $(40 \mathrm{~g}, 19 \times 240 \mathrm{~mm})$. The column was thoroughly washed with benzene and eluted with benzene- $\mathrm{CHCl}_{3}(1: 1)$. Fractions $(50 \mathrm{ml}$ each) were collected and monitored by silica gel TLC and ${ }^{1} \mathrm{H}-\mathrm{NMR}$ spectroscopy. Fractions $1-5$ afforded a colorless oil, (7Spaeonimetaboline I, 2, $26 \mathrm{mg}, 11 \%)^{2)}$ and fractions $11-15$ yielded a crystalline compound $(23 \mathrm{mg}, 9.6 \%)$, which gave pure crystals from hexane- $\mathrm{CHCl}_{3}(9: 1)(3,11 \mathrm{mg})$ on recrystallization. Fractions $6-10$ gave a mixture of 2 and 3 (21 mg, 8.8\%).

$7 S$-Paeonimetaboline I (2) - The physical properties were reported in the previous paper. ${ }^{2)}$

7R-Paeonimetaboline I (3)-Colorless prisms, mp 146-148 ${ }^{\circ} \mathrm{C}$. High resolution MS: Found, 198.0853; Calcd for $\mathrm{M}^{+}, \mathrm{C}_{10} \mathrm{H}_{14} \mathrm{O}_{4}, 198.0892$. IR $v_{\max }^{\mathrm{KBr}} \mathrm{cm}^{-1}: 3420(\mathrm{OH}), 1705(\mathrm{C}=\mathrm{O}) .{ }^{1} \mathrm{H}-\mathrm{NMR}\left(\mathrm{CDCl}_{3}, 400 \mathrm{MHz}\right) \delta: 0.90(3 \mathrm{H}, \mathrm{d}$, $\left.J=7.3 \mathrm{~Hz}, 8-\mathrm{H}_{3}\right), 1.29\left(3 \mathrm{H}, \mathrm{s}, 10-\mathrm{H}_{3}\right), 2.07(1 \mathrm{H}, \mathrm{dq}, J=7.5,7.3 \mathrm{~Hz}, 7-\mathrm{H}), 2.15$ and 2.35 (each $1 \mathrm{H}, \mathrm{dd}, J=13.4,2.3 \mathrm{~Hz}$; $\left.J=13.4,3.4 \mathrm{~Hz}, 5-\mathrm{H}_{2}\right), 2.60$ and $2.64\left(2 \mathrm{H}, \mathrm{ABq}, J=17.7 \mathrm{~Hz}, 2-\mathrm{H}_{2}\right), 2.65(1 \mathrm{H}, \mathrm{m}, 4-\mathrm{H}), 5.14(1 \mathrm{H}, \mathrm{brs}, 9-\mathrm{H}) . \mathrm{MS} m / z$ : $198\left(\mathrm{M}^{+}\right), 180\left(\mathrm{M}^{+}-\mathrm{H}_{2} \mathrm{O}\right), 152,124,109,98,83,69$ (base peak), 55. ${ }^{13} \mathrm{C}-\mathrm{NMR}$ : see Table I.

Metabolism of 1 by Bacteroides fragilis ss. thetaotus_-Compound $1(2.1 \mathrm{~g})$ was incubated with B. fragilis ss. thetaotus under conditions similar to those described above. After extraction with AcOEt, the organic layer was evaporated in vacuo to give an oily residue $(0.9 \mathrm{~g})$. The residue was chromatographed on silica gel $(80 \mathrm{~g}$; column size, $19 \times 350 \mathrm{~mm})$. The column was washed with benzene and eluted with benzene- $\mathrm{CHCl}_{3}(1: 1)$. Fractions were collected $(60 \mathrm{ml} /$ flask). Fractions $32-41,42-49$ and $50-61$ afforded 2 (colorless oil, $105 \mathrm{mg}, 12.6 \%$ ), a mixture of 2 and 3 (oil, $103 \mathrm{mg}, 12.3 \%$ ) and 3 (prisms, $52 \mathrm{mg}, 6.2 \%$ ), respectively. Another oily substance $(8 \mathrm{mg}, 0.9 \%)$ was obtained from a $\mathrm{CHCl}_{3}$ eluate; this was identical with paeonimetaboline II (4). ${ }^{2)}$

X-Ray Analysis of 3 - The unit-cell constants and intensities were measured at room temperature using $\mathrm{Ni}$ filtered $\mathrm{Cu} K_{\alpha}$ radiation on a Rigaku four-circle diffractometer. Crystal data are as follows: $\mathrm{C}_{10} \mathrm{H}_{14} \mathrm{O}_{4}, M_{\mathrm{r}}=198.1$, orthorhombic, space group $P 2_{1} 2_{1} 2_{1}, a=11.727(3), \quad b=7.463(2) . \quad c=10.852(3) \AA, \quad V=949.8 \AA^{3}, \quad Z=4, \quad D_{\mathrm{c}}=$ $1.385 \mathrm{~g} \cdot \mathrm{cm}^{-3}$. The $\theta-2 \theta$ scan technique was applied with scan width of $1.1^{\circ} \pm 0.15^{\circ} \tan \theta$. The backgrounds were counted for $4 \mathrm{~s}$ on both sides of the scan range. Of the 877 independent reflections measured up to $2 \theta=123^{\circ}, 851$ reflections with $F>3 \sigma(F)$ were used for the structure analysis. Periodically monitored reflections showed no significant change in intensity. The intensities were corrected for Lorentz and polarization factors. The structure was solved by the direct method. ${ }^{4)}$ The positional and anisotropic thermal parameters were refined by the block-diagonal least-squares method. ${ }^{5)}$ The hydrogen atoms were located from a difference Fourier synthesis, and included in the successive refinements with isotropic temperature factors. Extinction correction was made for the eight strongest reflections. The unit weight was applied for all reflections. The final $R$ value was 0.041 for 851 significant reflections. The atomic scattering factors were taken from the International Tables for X-Ray Crystallography. ${ }^{6}$

Paeonimetaboline II (4) _ Epimeric mixture consisting of $4 a(33 \%)$ and $4 b(67 \%)$. The following assignments of ${ }^{1} \mathrm{H}-\mathrm{NMR}$ signals $\left(\mathrm{CDCl}_{3}, 400 \mathrm{MHz}\right)$ were made on the basis of the peak intensities of paired signals. $4 \mathrm{a}: \delta: 1.12(3 \mathrm{H}$, $\left.\mathrm{d}, J=6.4 \mathrm{~Hz}, 8-\mathrm{H}_{3}\right), 1.36\left(3 \mathrm{H}, \mathrm{s}, 10-\mathrm{H}_{3}\right), 2.02(1 \mathrm{H}, \mathrm{ddq}, J=14.5,8.0,6.5 \mathrm{~Hz}, 7-\mathrm{H}), 2.25$ and $2.29(2 \mathrm{H}, \mathrm{ABq}$, $\left.J=12.8 \mathrm{~Hz}, 2-\mathrm{H}_{2}\right), c a .2 .27(1 \mathrm{H}, \mathrm{m}$, overlapped, $4-\mathrm{H}), 2.41$ and 2.85 (each $1 \mathrm{H}, \mathrm{d}$ and dd, $J=14.5 \mathrm{~Hz} ; J=14.5,7.4 \mathrm{~Hz}$, $\left.5-\mathrm{H}_{2}\right), 3.65$ and 4.05 (each $\left.1 \mathrm{H}, \mathrm{dd}, J=16.8,7.8 \mathrm{~Hz} ; J=16.8,8.0 \mathrm{~Hz}, 9-\mathrm{H}_{2}\right) .4 \mathrm{~b}: \delta: 1.13\left(3 \mathrm{H}, \mathrm{d}, J=6.4 \mathrm{~Hz}, 8-\mathrm{H}_{3}\right), 1.33$ $\left(3 \mathrm{H}, \mathrm{s}, 10-\mathrm{H}_{3}\right), 2.15(1 \mathrm{H}, \mathrm{m}, 7-\mathrm{H}), 2.32$ and $2.38\left(2 \mathrm{H}, \mathrm{ABq}, J=13.2 \mathrm{~Hz}, 2-\mathrm{H}_{2}\right), c a .2 .38(1 \mathrm{H}, \mathrm{m}$, overlapped, $4-\mathrm{H}), 2.37$ and 2.72 (each $1 \mathrm{H}, \mathrm{d}$ and dd, $J=14.0 \mathrm{~Hz} ; J=14.0,7.0 \mathrm{~Hz}, 5-\mathrm{H}_{2}$ ), 3.67 and 4.08 (each $1 \mathrm{H}, \mathrm{dd}, J=16.8,9.2 \mathrm{~Hz} ; J=16.8$, $\left.8.7 \mathrm{~Hz}, 9-\mathrm{H}_{2}\right)$.

7S-Paeonimetaboline-6-O-3,5-dinitrophenyl Carbamate (5)—3,5-Dinitrophenyl isocyanate (25 mg, Sumitomo Chem. Co. Ltd., Osaka) and dry pyridine $(0.05 \mathrm{ml})$ were added to a solution of $2(3.0 \mathrm{mg})$ in dry toluene $(0.5 \mathrm{ml})$. The mixture was kept overnight at $37^{\circ} \mathrm{C}$, and then cooled. Methanol $(2 \mathrm{ml})$ was added and the solvent was removed in vacuo. The product was subjected to preparative silica gel TLC to give a pure carbamate $(5, \mathrm{ca} .2 \mathrm{mg})$ as a colorless oil. 
${ }^{1} \mathrm{H}-\mathrm{NMR}\left(\mathrm{CDCl}_{3}, 270 \mathrm{MHz}\right) \delta: 1.18\left(3 \mathrm{H}, \mathrm{d}, J=7.3 \mathrm{~Hz}, 8-\mathrm{H}_{3}\right), 1.37\left(3 \mathrm{H}, \mathrm{s}, 10-\mathrm{H}_{3}\right), 1.99(1 \mathrm{H}$, br q, $J=7.3 \mathrm{~Hz}, 7-\mathrm{H})$, $2.56(1 \mathrm{H}, \mathrm{m}, 4-\mathrm{H}), 2.71$ and $2.79\left(\right.$ each $\left.1 \mathrm{H}, \mathrm{dd}, J=13.6,3.4 \mathrm{~Hz} ; J=13.6,2.7 \mathrm{~Hz}, 5-\mathrm{H}_{2}\right), 2.81$ and $3.28($ each $1 \mathrm{H}, \mathrm{d}$, $\left.J=17.3 \mathrm{~Hz}, 2-\mathrm{H}_{2}\right), 5.31(1 \mathrm{H}$, br s, $9-\mathrm{H}), 8.67\left(2 \mathrm{H}, \mathrm{d}, J=1.9 \mathrm{~Hz}, 2^{\prime}-, 6^{\prime}-\mathrm{H}\right), 8.78\left(1 \mathrm{H}, \mathrm{t}, J=1.9 \mathrm{~Hz}, 4^{\prime}-\mathrm{H}\right)$.

7R-Paeonimetaboline-6-O-3,5-dinitrophenyl Carbamate (6)-Under conditions similar to those described above, 3 was reacted with 3,5-dinitrophenyl isocyanate to yield 6 as a white powder. ${ }^{1} \mathrm{H}-\mathrm{NMR}\left(\mathrm{CDCl}_{3}, 270 \mathrm{MNz}\right) \delta$ : $0.93\left(3 \mathrm{H}, \mathrm{d}, J=7.3 \mathrm{~Hz}, 8-\mathrm{H}_{3}\right), 1.37\left(3 \mathrm{H}, \mathrm{s}, 10-\mathrm{H}_{3}\right), 2.18(1 \mathrm{H}, \mathrm{dq}, J=7.5,7.3 \mathrm{~Hz}, 7-\mathrm{H}), 2.61$ and $2.85($ each $1 \mathrm{H}, \mathrm{dd}$, $\left.J=13.2,3.3 \mathrm{~Hz} ; J=13.2,2.3 \mathrm{~Hz}, 5-\mathrm{H}_{2}\right), 2.71(1 \mathrm{H}, \mathrm{m}, 4-\mathrm{H}), 2.75$ and $3.14\left(\right.$ each $\left.1 \mathrm{H}, \mathrm{d}, J=17.6 \mathrm{~Hz}, 2-\mathrm{H}_{2}\right), 5.32(1 \mathrm{H}$, br s, 9-H), $8.67\left(2 \mathrm{H}, \mathrm{d}, J=1.9 \mathrm{~Hz}, 2^{\prime}-, 6^{\prime}-\mathrm{H}\right), 8.78\left(1 \mathrm{H}, \mathrm{t}, J=1.9 \mathrm{~Hz}, 4^{\prime}-\mathrm{H}\right)$.

5-Hydroxy-3,6-dimethyl-2,3-dihydrobenzofuran (7)—Colorless oil; $[\alpha]_{\mathrm{D}}^{24}: 0(c=0.22, \mathrm{MeOH})$. High resolution MS: Found 164.0847; Calcd for $\mathrm{M}^{+}, \mathrm{C}_{10} \mathrm{H}_{12} \mathrm{O}_{2}, 164.0837$. MS $m / z: 164\left(\mathrm{M}^{+}\right), 159\left(\mathrm{M}^{+}-\mathrm{CH}_{3}\right), 121,91$ (base peak), 77. $\mathrm{UV} \lambda{ }_{\max }^{\mathrm{EtOH}} \mathrm{nm}(\log \varepsilon): 319$ (2.70), $313 \mathrm{sh}(2.65) . \mathrm{IR} v_{\max }^{\mathrm{KBr}} \mathrm{cm}^{-1}: 3360,1458,1165,865 .{ }^{1} \mathrm{H}-\mathrm{NMR}\left(\mathrm{CDCl}_{3}, 270 \mathrm{MHz}\right) \delta$ : $1.21(3 \mathrm{H}, \mathrm{d}, J=7.0 \mathrm{~Hz}, 3-\mathrm{Me}), 2.12(3 \mathrm{H}, \mathrm{s}, 6-\mathrm{Me}), 3.41(1 \mathrm{H}$, br sextet, $J=7.4 \mathrm{~Hz}, 3-\mathrm{H}), 3.94(1 \mathrm{H}, \mathrm{dd}, J=8.7,7.4 \mathrm{~Hz}, 2-$ $\left.\mathrm{H}_{\mathrm{a}}\right), 4.52\left(1 \mathrm{H}, \mathrm{dd}, J=8.7,8.5 \mathrm{~Hz}, 2-\mathrm{H}_{\mathrm{b}}\right), 6.49$ and $6.54($ each $1 \mathrm{H}, \mathrm{s}, 4-\mathrm{H}, 7-\mathrm{H}) .{ }^{13} \mathrm{C}-\mathrm{NMR}\left(\mathrm{CDCl}_{3}, 22.5 \mathrm{MHz}\right) \delta: 16.0$ (q, 3-Me), 19.1 (q, 6-Me), 36.8 (d, C-3), 78.3 (t,C-2), 110.0 (d), 110.8 (d), 122.9 (s, C-6), 130.6 (s), 147.7 (s), 153.6 (s).

5-Acetoxy-3,6-dimethyl-2,3-dihydrobenzofuran (8)—On acetylation of $7(4 \mathrm{mg})$ with acetic anhydride $(0.3 \mathrm{ml})$ in pyridine $(0.3 \mathrm{ml})$, a colorless oil $(\mathbf{8}, 2 \mathrm{mg})$ was obtained. MS $m / z: 206\left(\mathrm{M}^{+}\right), 164,149$ (base peak), 121, 83, 73, 57, 55. ${ }^{1} \mathrm{H}-\mathrm{NMR}\left(\mathrm{CDCl}_{3}, 270 \mathrm{MHz}\right) \delta: 1.22(3 \mathrm{H}, \mathrm{d}, J=6.9 \mathrm{~Hz}, 3-\mathrm{Me}), 2.04(3 \mathrm{H}, \mathrm{s}, 6-\mathrm{Me}), 2.22(3 \mathrm{H}, \mathrm{s}, 5-\mathrm{OAc}), 3.45(1 \mathrm{H}, \mathrm{br}$ sextet, $J=7.5 \mathrm{~Hz}, 3-\mathrm{H}), 3.99\left(1 \mathrm{H}, \mathrm{dd}, J=8.6,7.6 \mathrm{~Hz}, 2-\mathrm{H}_{\mathrm{a}}\right), 4.60\left(1 \mathrm{H}, \mathrm{dd}, J=8.6,8.4 \mathrm{~Hz}, 2-\mathrm{H}_{\mathrm{b}}\right), 6.55(1 \mathrm{H}, \mathrm{s}, 7-\mathrm{H}), 6.71$ $(1 \mathrm{H}, \mathrm{s}, 4-\mathrm{H})$.

\section{Results}

Metabolism of Paeoniflorin (1) by Bacteroides fragilis ss. thetaotus and Lactobacillus brevis

Through a $4 \mathrm{~h}$ anaerobic incubation with a bacterial suspension of either $B$. fragilis or $L$. brevis, 1 was converted to two major metabolites ( 2 and 3 ), which were not well separated by TLC, together with two minor metabolites (4a and $\mathbf{4 b})$ in the case of the former bacterium. These metabolites decreased in amount during prolonged incubation and disappeared almost completely in $16 \mathrm{~h}$ under the incubation conditions used. Bacteroides fragilis seemed to grow more rapidly and to have stronger metabolizing activity than L. brevis.

\section{Structures of Metabolites}

The first major metabolite ( $R f=0.52$ on TLC) was identified by direct spectroscopic comparisons as $7 S$-paeonimetaboline I (2) which had previously been obtained by incubation of 1 with a mixture of bacteria from human feces.

The second major metabolite, colorless prisms, mp $146-148^{\circ} \mathrm{C}$, had almost the same $R f$ value with as 2 , but could be separated by repeated column chromatography. The chemical composition was determined to be $\mathrm{C}_{10} \mathrm{H}_{14} \mathrm{O}_{4}$, isomeric with 2 , by high-resolution mass spectrometry. The compound showed no UV absorption, but there were characteristic strong IR bands assignable to a hydroxyl $\left(3420 \mathrm{~cm}^{-1}\right)$ and a ketonic carbonyl $\left(1705 \mathrm{~cm}^{-1}\right)$ in a six-

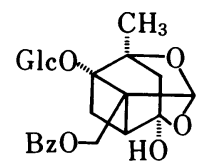

1<smiles>CC1CC(=O)C(C)(O)C(O)C1CO</smiles>

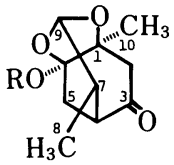

2: $\mathrm{R}=-\mathrm{H}$

$5: \mathrm{R}=-\mathrm{CO}-\mathrm{NH}-\mathrm{C}_{6} \mathrm{H}_{3}-\left(\mathrm{NO}_{2}\right)_{2}$<smiles>C[C@H]1CC(=O)[C@@](C)(O)C[C@H]1OC(=O)O</smiles>

10



$3: \mathrm{R}_{1}=-\mathrm{H}, \mathrm{R}_{2}=-\mathrm{H}$

$6: \mathrm{R}_{1}=-\mathrm{CO}-\mathrm{NH}-\mathrm{C}_{6} \mathrm{H}_{3}-\left(\mathrm{NO}_{2}\right)_{2}, \mathrm{R}_{2}=-\mathrm{H}$

$9: \mathrm{R}_{1}=-\mathrm{H}, \mathrm{R}_{2}=-\mathrm{O}-\mathrm{CO}-\mathrm{C}_{6} \mathrm{H}_{5}$<smiles>[R20]c1cc2c(cc1C)OCC2C</smiles>

$7: \mathrm{R}=-\mathrm{H}$

$8: \mathrm{R}=-\mathrm{CO}-\mathrm{CH}_{3}$

Chart 1. Structures of Paeoniflorin, Paeonimetabolines and Related Compounds 


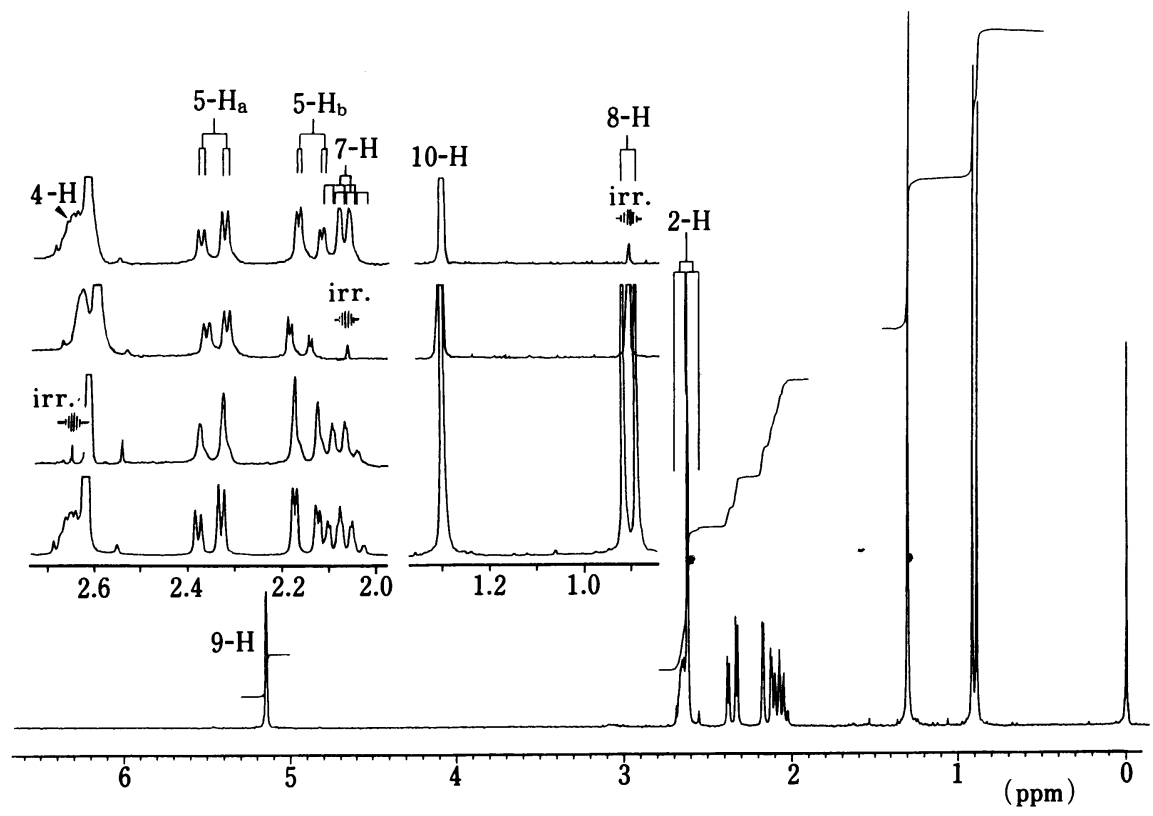

Fig. 1. ${ }^{1} \mathrm{H}-\mathrm{NMR}$ Spectrum of $7 R$-Paeonimetaboline I (3) Measured at $270 \mathrm{MHz}$

TABlE I. ${ }^{13}$ C-NMR Data for Paeonimetabolines and Related Compounds

\begin{tabular}{|c|c|c|c|c|c|}
\hline \multirow{2}{*}{ Carbon No. } & \multicolumn{5}{|c|}{ Compounds } \\
\hline & 2 & 3 & $4 a^{a)}$ & $4 b^{a)}$ & 10 \\
\hline C-1 & $77.4(\mathrm{~s})$ & $78.3(\mathrm{~s})$ & $72.8(\mathrm{~s})$ & $72.8(\mathrm{~s})$ & $73.6(\mathrm{~s})$ \\
\hline C-2 & $47.5(t)$ & $46.8(t)$ & $38.6(t)$ & $38.8(t)$ & $35.5(t)$ \\
\hline$C-3$ & $210.9(\mathrm{~s})$ & $210.1(\mathrm{~s})$ & $104.8(\mathrm{~s})$ & $104.8(\mathrm{~s})$ & 73.5 (d) \\
\hline$C-4$ & $50.2(\mathrm{~d})$ & 49.6 (d) & $52.3(\mathrm{~d})$ & $53.4(d)$ & 37.9 (d) \\
\hline C-5 & $30.4(t)$ & $34.3(\mathrm{t})$ & $47.5(\mathrm{t})$ & $47.1(\mathrm{t})$ & $42.1(t)$ \\
\hline C-6 & $101.6(\mathrm{~s})$ & $101.3(\mathrm{~s})$ & $212.7(\mathrm{~s})$ & $212.7(\mathrm{~s})$ & $210.4(s)$ \\
\hline $\mathrm{C}-7$ & 37.9 (d) & 38.4 (d) & 40.2 (d) & 42.1 (d) & $43.8(d)$ \\
\hline C-8 & $14.6(q)$ & $13.3(\mathrm{q})$ & $16.3(q)$ & $15.7(q)$ & $13.1(\mathrm{q})$ \\
\hline C-9 & $103.1(\mathrm{~d})$ & 103.0 (d) & $72.4(\mathrm{t})$ & $73.4(t)$ & $177.0(\mathrm{~s})$ \\
\hline C- 10 & $20.9(q)$ & $21.1(\mathrm{q})$ & $25.2(\mathrm{q})$ & $25.6(\mathrm{q})$ & $24.8(\mathrm{q})$ \\
\hline
\end{tabular}

a) $4 \mathrm{a}$ and $4 \mathrm{~b}$ were not separated, but their ${ }^{13} \mathrm{C}-\mathrm{NMR}$ chemical shifts were deduced on the basis of the relative signal intensities. Abbreviations given in parentheses indicate the signal patterns based on OFR and INEPT experiments. s, singlet; $d$, doublet; $t$, triplet; $q$, quartet.

membered ring. The ${ }^{1} \mathrm{H}-\mathrm{NMR}$ (Fig. 1) and ${ }^{13} \mathrm{C}-\mathrm{NMR}$ ('Table I) spectra showed the presence of identical functional groups with those of 2 ; a sec-methyl $\left({ }^{1} \mathrm{H}, \delta 0.90 ;{ }^{13} \mathrm{C}, \delta 13.3\right)$, a tertmethyl $\left({ }^{1} \mathrm{H}, \delta 1.29 ;{ }^{13} \mathrm{C}, \delta 21.1\right)$, an acetal $\left({ }^{1} \mathrm{H}, \delta 5.14 ;{ }^{13} \mathrm{C}, \delta 103.0\right)$, a hemiketal $\left({ }^{13} \mathrm{C}, \delta 101.3\right)$, a ketonic carbonyl $\left({ }^{13} \mathrm{C}, \delta 210.1\right)$, etc. The ${ }^{1} \mathrm{H}$-double resonance experiments (Fig. 1) indicated the following spin correlations: a methine proton at $\delta 2: 65(\mathrm{~m}, 4-\mathrm{H})$ versus methylene protons at $\delta 2.15$ and 2.35 (each dd, $5-\mathrm{H}_{\mathrm{a}}$ and $5-\mathrm{H}_{\mathrm{b}}$ ) or a methine proton at $\delta 2.07(\mathrm{dq}, 7-\mathrm{H})$; a methine proton $(7-\mathrm{H})$ versus methyl protons $\left(8-\mathrm{H}_{3}\right)$ at $\delta 0.90$. These findings suggested that 3 had the same skeleton as 2 , which was further supported by the evidence that most of the ${ }^{13} \mathrm{C}$-signals of 3 were quite similar to those of 2, except the C-8 and C-5 signals. In contrast with the case 
of 2 , in which $\gamma$-gauche steric interaction is apparent between the C-8 and C-5 carbons, no appreciable neighboring group effect was observed in $\mathbf{3}$, based on a comparison of the signals with the corresponding signals of paeoniflorigenone (9). ${ }^{7}$ This suggests that the C-8 methyl group is projected just above the $\mathrm{C}-3$ carbonyl group (7R-configuration), as in 9 . This conclusion is further supported by the observation of an upfield shift (ca. $0.22 \mathrm{ppm}$ unit) of the C-8 methyl proton signal in 3 compared to that of $\mathbf{2}$ in the ${ }^{1} \mathrm{H}-\mathrm{NMR}$ spectrum. Based on the above evidence, 3 was deduced to be $7 R$-paeonimetaboline I (Chart 1).

The structure and stereochemistry were finally established by a single-crystal X-ray analysis using the direct method. A perspective view of the molecule drawn by DCMS- $3^{8)}$ is shown in Fig. 2. The atomic parameters, ${ }^{9)}$ bond lengths and bond angles are listed in Tables II and III.



Fig. 2. Perspective View of $7 R$-Paeonimetaboline I (3)

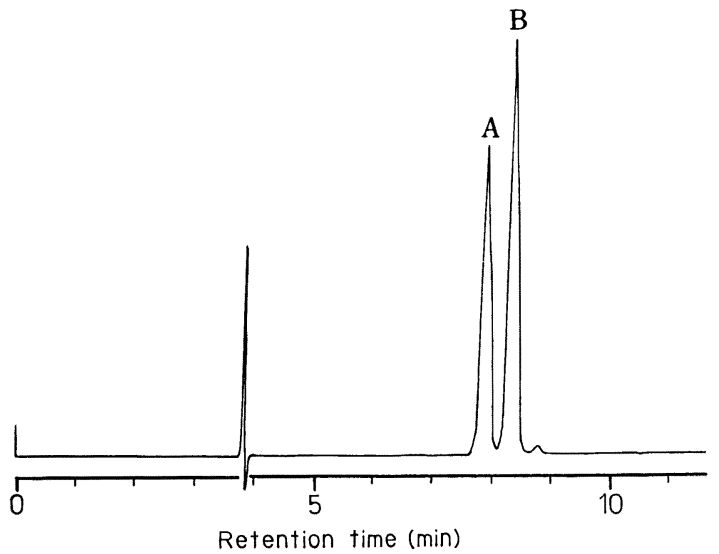

Fig. 3. HPLC Elution Profile of Carbamates of the $7 R$ and $7 S$ Epimers of Paeonimetaboline $I$

Conditions: column, Nucleosil $50-5(5 \mu, 4.6 \mathrm{~mm}$ i.d. $\times 25 \mathrm{~cm})$; mobile phase, $n$-hexane-1,2-dichloroethane-ethanol $(20: 4: 1)$; flow rate, $1.3 \mathrm{ml} / \mathrm{min}$; pressure, $30 \mathrm{~kg} / \mathrm{cm}^{2}$; detection, UV at $250 \mathrm{~nm}$. Peaks A and $\mathbf{B}$ are the 3,5-dinitrophenylcarbamates of $\mathbf{2}$ and $\mathbf{3}$, respectively.

TABLE II: Final Atomic Coordinates of $7 R$-Paeonimetaboline I (3)

\begin{tabular}{cccccccc}
\hline \hline $\begin{array}{c}\text { Non-hydrogen } \\
\text { atoms }\end{array}$ & $x$ & $y$ & $z$ & $\begin{array}{c}\text { Hydrogen } \\
\text { atoms }\end{array}$ & $x$ & $y$ & $z$ \\
\hline $\mathrm{C}(1)$ & $0.3634(4)$ & $0.6206(6)$ & $0.3405(4)$ & $\mathrm{H}(1)$ & $0.406(4)$ & $0.613(6)$ & $0.243(4)$ \\
$\mathrm{C}(2)$ & $0.3976(3)$ & $0.5368(5)$ & $0.5412(3)$ & $\mathrm{H}(2)$ & $0.478(4)$ & $0.725(6)$ & $0.666(4)$ \\
$\mathrm{C}(3)$ & $0.3914(4)$ & $0.6883(6)$ & $0.6361(4)$ & $\mathrm{H}(3)$ & $0.359(4)$ & $0.649(7)$ & $0.713(4)$ \\
$\mathrm{C}(4)$ & $0.3342(4)$ & $0.8491(6)$ & $0.5783(4)$ & $\mathrm{H}(4)$ & $0.174(4)$ & $0.913(6)$ & $0.483(4)$ \\
$\mathrm{C}(5)$ & $0.2428(4)$ & $0.8062(6)$ & $0.4834(4)$ & $\mathrm{H}(5)$ & $0.153(4)$ & $0.632(6)$ & $0.610(4)$ \\
$\mathrm{C}(6)$ & $0.1881(3)$ & $0.6262(6)$ & $0.5178(4)$ & $\mathrm{H}(6)$ & $0.116(4)$ & $0.596(6)$ & $0.462(4)$ \\
$\mathrm{C}(7)$ & $0.2752(3)$ & $0.4806(6)$ & $0.4956(4)$ & $\mathrm{H}(7)$ & $0.227(4)$ & $0.786(7)$ & $0.295(4)$ \\
$\mathrm{C}(8)$ & $0.2945(4)$ & $0.7940(6)$ & $0.3519(4)$ & $\mathrm{H}(8)$ & $0.452(4)$ & $0.961(7)$ & $0.365(4)$ \\
$\mathrm{C}(9)$ & $0.3670(5)$ & $0.9562(7)$ & $0.3150(4)$ & $\mathrm{H}(9)$ & $0.328(4)$ & $1.069(7)$ & $0.333(4)$ \\
$\mathrm{C}(10)$ & $0.4698(4)$ & $0.3824(6)$ & $0.5846(5)$ & $\mathrm{H}(10)$ & $0.388(4)$ & $0.954(7)$ & $0.220(5)$ \\
$\mathrm{O}(1)$ & $0.2943(3)$ & $0.4689(4)$ & $0.3641(2)$ & $\mathrm{H}(11)$ & $0.553(4)$ & $0.419(6)$ & $0.602(4)$ \\
$\mathrm{O}(2)$ & $0.4501(2)$ & $0.6118(4)$ & $0.4317(2)$ & $\mathrm{H}(12)$ & $0.478(4)$ & $0.283(7)$ & $0.518(5)$ \\
$\mathrm{O}(3)$ & $0.3584(3)$ & $0.9998(4)$ & $0.6086(3)$ & $\mathrm{H}(13)$ & $0.452(4)$ & $0.328(7)$ & $0.647(4)$ \\
$\mathrm{O}(4)$ & $0.2330(2)$ & $0.3200(4)$ & $0.5396(3)$ & $\mathrm{H}(14)$ & $0.283(4)$ & $0.251(6)$ & $0.537(4)$ \\
\hline
\end{tabular}


TABLE III. Bond Lengths $(\AA)$ and Angles $\left({ }^{\circ}\right)$ for Non-hydrogen Atoms of $7 R$-Paeonimetaboline I (3)

\begin{tabular}{lrllll}
\hline \hline $\mathrm{C}(1)-\mathrm{C}(8)$ & $1.530(6)$ & $\mathrm{C}(1)-\mathrm{O}(1)$ & $1.416(6)$ & $\mathrm{C}(1)-\mathrm{O}(2)$ & $1.420(5)$ \\
$\mathrm{C}(2)-\mathrm{C}(3)$ & $1.532(6)$ & $\mathrm{C}(2)-\mathrm{C}(7)$ & $1.576(6)$ & $\mathrm{C}(2)-\mathrm{C}(10)$ & $1.506(6)$ \\
$\mathrm{C}(2)-\mathrm{O}(2)$ & $1.450(5)$ & $\mathrm{C}(3)-\mathrm{C}(4)$ & $1.512(6)$ & $\mathrm{C}(4)-\mathrm{C}(5)$ & $1.520(6)$ \\
$\mathrm{C}(4)-\mathrm{O}(3)$ & $1.206(6)$ & $\mathrm{C}(5)-\mathrm{C}(6)$ & $1.535(6)$ & $\mathrm{C}(5)-\mathrm{C}(8)$ & $1.553(6)$ \\
$\mathrm{C}(6)-\mathrm{C}(7)$ & $1.510(6)$ & $\mathrm{C}(7)-\mathrm{O}(1)$ & $1.447(5)$ & $\mathrm{C}(7)-\mathrm{O}(4)$ & $1.382(5)$ \\
$\mathrm{C}(8)-\mathrm{C}(9)$ & $1.532(7)$ & & & & \\
$\mathrm{C}(8)-\mathrm{C}(1)-\mathrm{O}(1)$ & $111.1(4)$ & $\mathrm{C}(8)-\mathrm{C}(1)-\mathrm{O}(2)$ & $111.1(4)$ \\
$\mathrm{O}(1)-\mathrm{C}(1)-\mathrm{O}(2)$ & $104.3(3)$ & $\mathrm{C}(3)-\mathrm{C}(2)-\mathrm{C}(7)$ & $111.4(3)$ \\
$\mathrm{C}(3)-\mathrm{C}(2)-\mathrm{C}(10)$ & $112.4(4)$ & $\mathrm{C}(3)-\mathrm{C}(2)-\mathrm{O}(2)$ & $106.6(3)$ \\
$\mathrm{C}(7)-\mathrm{C}(2)-\mathrm{C}(10)$ & $114.0(3)$ & $\mathrm{C}(7)-\mathrm{C}(2)-\mathrm{O}(2)$ & $103.4(3)$ \\
$\mathrm{C}(10)-\mathrm{C}(2)-\mathrm{O}(2)$ & $108.3(3)$ & $\mathrm{C}(2)-\mathrm{C}(3)-\mathrm{C}(4)$ & $109.2(4)$ \\
$\mathrm{C}(3)-\mathrm{C}(4)-\mathrm{C}(5)$ & $115.3(4)$ & $\mathrm{C}(3)-\mathrm{C}(4)-\mathrm{O}(3)$ & $121.5(4)$ \\
$\mathrm{C}(5)-\mathrm{C}(4)-\mathrm{O}(3)$ & $123.2(4)$ & $\mathrm{C}(4)-\mathrm{C}(5)-\mathrm{C}(6)$ & $108.3(4)$ \\
$\mathrm{C}(4)-\mathrm{C}(5)-\mathrm{C}(8)$ & $111.1(4)$ & $\mathrm{C}(6)-\mathrm{C}(5)-\mathrm{C}(8)$ & $109.6(4)$ \\
$\mathrm{C}(5)-\mathrm{C}(6)-\mathrm{C}(7)$ & $108.0(4)$ & $\mathrm{C}(2)-\mathrm{C}(7)-\mathrm{C}(6)$ & $112.0(3)$ \\
$\mathrm{C}(2)-\mathrm{C}(7)-\mathrm{O}(1)$ & $100.6(3)$ & $\mathrm{C}(2)-\mathrm{C}(7)-\mathrm{O}(4)$ & $116.6(3)$ \\
$\mathrm{C}(6)-\mathrm{C}(7)-\mathrm{O}(1)$ & $107.8(3)$ & $\mathrm{C}(6)-\mathrm{C}(7)-\mathrm{O}(4)$ & $109.1(3)$ \\
$\mathrm{O}(1)-\mathrm{C}(7)-\mathrm{O}(4)$ & $110.1(3)$ & $\mathrm{C}(1)-\mathrm{C}(8)-\mathrm{C}(5)$ & $109.2(4)$ \\
$\mathrm{C}(1)-\mathrm{C}(8)-\mathrm{C}(9)$ & $110.8(4)$ & $\mathrm{C}(5)-\mathrm{C}(8)-\mathrm{C}(9)$ & $114.2(4)$ \\
$\mathrm{C}(1)-\mathrm{O}(1)-\mathrm{C}(7)$ & $102.6(3)$ & $\mathrm{C}(1)-\mathrm{O}(2)-\mathrm{C}(2)$ & $106.6(3)$ \\
\hline
\end{tabular}

Despite many attempts, we could not quantitatively analyze the two epimers by TLCdensitometry or GC-MS after derivatization (silylation), but the 3,5-dinitrophenylcarbamates of 2 and 3 were well separated with high sensitivity by normal phase HPLC. Figure 3 shows a chromatogram of the mixture; peaks $\mathrm{A}$ and $\mathrm{B}$ were assigned to the 3,5-dinitrophenylcarbamates of $\mathbf{2}$ and $\mathbf{3}$, respectively.

The minor metabolites $(R f=0.42)$ were obtained as a colorless oil, identical with paeonimetaboline II, as had been reported in the previous paper. ${ }^{2)}$ The oil, however, was a mixture of isomers (4a and $4 \mathbf{b}$ ) in a ratio of $2: 1$ on the basis of $400 \mathrm{MHz}{ }^{1} \mathrm{H}-\mathrm{NMR}$ analysis. Since various attempts to separate the two isomers were unsuccessful due to their extreme instability, the mixture was directly analyzed.

The ${ }^{1} \mathrm{H}-\mathrm{NMR}$ and ${ }^{13} \mathrm{C}$-NMR spectra (Table I) showed pairs of signals with different intensities, which may be assignable to sec-and tert-methyls, three methylenes including an oxymethylene, two methines including a methine adjacent to the sec-methyl, and hemiketal and ketonic carbons, but they did not exhibit either characteristic signals of glucose and benzoyl moieties present in the original substrate (1) or those of an acetal group present in the major metabolites ( 2 and 3 ). In addition, a significant difference in chemical shift (1.9 ppm) was only observed for the methyl-bearing carbon (C-7) between $\mathbf{4 a}$ and $\mathbf{4 b}$, suggesting a mixture of $7 R$ and $7 S$ epimers. Based on the above data and the chemical finding that the mixture were readily decomposed to 5-hydroxy-3,6-dimethyl-2,3-dihydrobenzofuran (7), as will be mentioned later, the structures of the epimers were proposed to be $4^{10}$ ) in Chart 1 .

When kept in $\mathrm{CHCl}_{3}-\mathrm{MeOH}$ for several days, the mixture of $\mathbf{4 a}$ and $\mathbf{4 b}$ decomposed to several products with UV absorption. The major product had $R f 0.71$, but was readily transformed to another compound (7) with $R f 0.59$. The isolation of the former compound was unsuccessful because of its extreme instability during purification, but the latter (7) was obtained as an oil with the molecular formula $\mathrm{C}_{10} \mathrm{H}_{12} \mathrm{O}_{2}$, which afforded a monoacetate on acetylation. The UV spectrum $\left(\lambda_{\max } 319 \mathrm{~nm}, \lambda_{\mathrm{sh}} 313 \mathrm{~nm}\right)$ showed the presence of a $p$ hydroquinone system ${ }^{11)}$ and the IR spectrum showed a strong absorption band due to a hydroxyl group. The ${ }^{1} \mathrm{H}-\mathrm{NMR}$ and ${ }^{13} \mathrm{C}-\mathrm{NMR}$ spectral evidence finally led to the structure of 
5-hydroxy-3,6-dimethyl-2,3-dihydrobenzofuran for 7.

\section{Discussion}

By anaerobic incubation with B. fragilis and L. brevis, paeoniflorin (1) was converted into $7 S$ and $7 R$ isomers of paeonimetaboline I ( 2 and 3 , respectively), as well as those of paeonimetaboline II (4a and $\mathbf{4 b}$ ) in the case of the former bacterium. In the transformation of 1 by the two bacterial strains, the newly isolated metabolite, 3 , seems to be formed through metabolic processes analogous to those involved in the case of 2 , as reported in the preceding paper (Chart 2); ${ }^{2}$ enzymic hydrolysis of glucosyl and benzoyl groups is considered to be the first step, followed by cleavages of the hemiketal-acetal linkage and the four-membered ring (i and ii), then formation of an aldehyde intermediate (iii) and cyclization to yield a hemiketalacetal derivative (iv). Non-stereospecific reduction of iv leads to a mixture of the $7 R$ and $7 S$ isomers (3 and 2). Since no conversion from $2 / 3$ to $\mathbf{4 a} / \mathbf{4 b}$, or vice versa, could be demonstrated during anaerobic incubation with either $B$. fragilis of $L$. brevis, the formation of 4 proceeds via an aldehyde intermediate (iii) and its reduced product (v). The alcohol (v) might cyclize to form a hemiketal at the C-3 position (vi), followed by non-stereospecific reduction of the terminal double bond to yield the $7 R$ and $7 S$ isomers of paeonimetaboline II (4a and $\mathbf{4 b})$. This mixture was readily dehydrated, followed by aromatization to yield a dihydrobenzofuran derivative (7).

7S-Paeonimetaboline I (2) has an appreciable suppressing effect on convulsions induced by pentylenetetrazole in rats. ${ }^{12)}$ Further studies are in progress to examine the difference in biological potency between the two epimers.
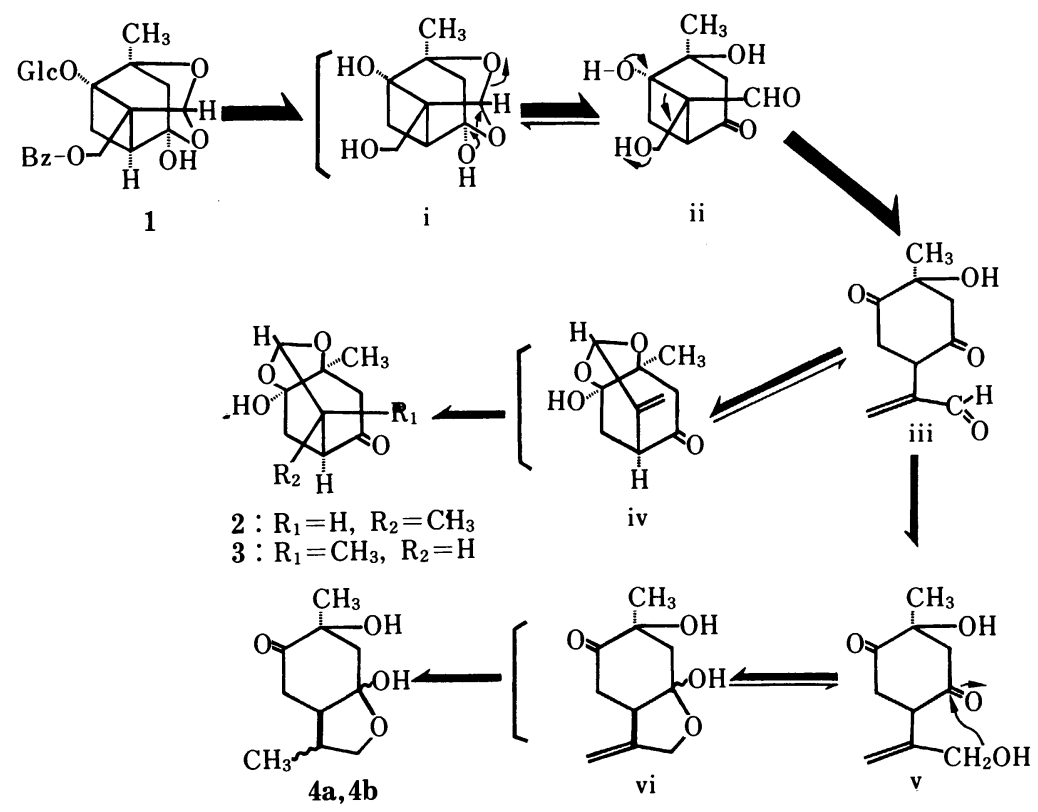

Chart 2. Possible Metabolic Processes of Paeoniflorin (1) by Intestinal Bacteria

Acknowledgement We are grateful to Professor Tohru Kikuchi (Research Institute for Wakan-Yaku, Toyama Medical and Pharmaceutical University) for providing facilities for $400 \mathrm{MHz}{ }^{1} \mathrm{H}-\mathrm{NHR}$ spectral measurements and to the staff of the Crystallographic Research Center of Osaka University for use of the four-circle diffractometer. This study was funded in part by Tsumura Juntendo Co. Ltd. (Tokyo). 


\section{References and Notes}

1) A part of this study was presented at the 33rd Annual Meeting of the Pharmacognostical Society of Japan, Saitama, 1986, Abstracts of Papers, p. 80.

2) M. Hattori, Y. Z. Shu, M. Shimizu, T. Hayashi, N. Morita, K. Kobashi, G. J. Xu and T. Namba, Chem. Pharm. Bull., 33, 3838 (1985).

3) M. Hattori, Y. Z. Shu, K. Kobashi and T. Namba, J. Med. Pharm. Soc. Wakan-Yaku, 2, 398 (1985).

4) G. Germain, P. Main and M. M. Woolfson, Acta Crystallogr., Sect. A, 27, 368 (1971).

5) T. Ashida, HBLS-V, The Universal Crystallographic Computing System-Osaka, The Computation Center of Osaka University, 1973, pp. 55-61.

6) “International Tables for X-Ray Crystallography," Vol. IV, Kynoch Press, Birmingham, 1974.

7) M. Shimizu, T. Hayashi, N. Morita, F. Kiuchi, H. Noguchi, Y. Iitaka and U. Sankawa, Chem. Pharm. Bull., 31, 577 (1983).

8) A. Takenaka, DCMS-3, Tokyo Institute of Technology, 1977.

9) The anisotropic temperature factors and a list of observed and calculated structure factors $(\times 10)$ are available from one of the authors (T.T.) on request.

10) In a comparison of the ${ }^{13} \mathrm{C}-\mathrm{NMR}$ spectra (Table I) of the mixture of $4 \mathbf{a}$ and $4 \mathbf{b}$ with those of 2,3 and paeonilactone $\mathbf{A}(\mathbf{1 0}),{ }^{3)}$ the $\mathrm{C}_{1}$ signal $(\delta 72.8)$ of either $\mathbf{4 a}$ or $\mathbf{4 b}$ appeared at a position similar to that in 10 ( $\delta 73.6)$ but not in 2 or 3 ( $\delta 77.4$ and 78.3), indicating that the ketonic carbonyl of both $4 \mathrm{a}$ and $\mathbf{4 b}$ is located at C6 but not C-3.

11) A. I. Scott, "Interpretation of the Ultra-Violet Spectra of Natural Products," Pergamon Press Ltd., London, 1962, pp. 91-97.

12) a) T. Namba, M. Hattori, Y. Z. Shu, A. Ishige, A. Sugimoto, K. Sekiguchi, M. Aburada and H. Hosoya, Abstracts of Papers, 106th Annual Meeting of the Pharmaceutical Society of Japan, Chiba, 1986, p. 214; $b$ ) Y. Z. Shu, M. Hattori, T. Namba, K. Mibu, T. Akao and K. Kobashi, Abstracts of Papers, 6th Symposium on the Development and Application of Naturally Occurring Drug Materials, Nagoya, 1986, p. 49; c) Idem, J. Pharmacobio-Dyn., 10, s-58 (1987). 